\title{
A 22-year-old Woman With SySTemic Lupus Erythematosus Develops Cardiac Tamponade
}

Brooks Kuhn, MS-III, Arthi Reddy, MD, Sorin Lazar, MD

\section{Introduction}

Systemic lupus erythematosus (SLE) is a common cause of pericardial effusion and acute pericarditis, but very rarely it can cause cardiac tamponade. ${ }^{1}$ We describe the case of a young female with SLE who developed cardiac tamponade after finishing treatment for acute pericarditis with a small pericardial effusion.

\section{Case Report}

A 22-year-old college student at a local university with a history of SLE diagnosed in 2003 (ANA $^{+}$, anti-dsDNA ${ }^{+}$, and anti-SS$\mathrm{A}^{+}$) presented to an outside hospital complaining of shortness of breath, fever, and pleuritic chest pain. These symptoms began acutely in the presence of a week-old lupus flare characterized by a butterfly malar rash and arthralgias. A chest CT demonstrated a small pericardial effusion without evidence of tamponade. She was diagnosed with pericarditis and subsequently her outpatient dose of prednisone was increased from $15 \mathrm{mg}$ /day to $60 \mathrm{mg} /$ day. However, she showed little clinical or radiographic improvement on CT. She was then scheduled to receive pulse dose steroids (methylprednisolone) for 3 days.

The patient presented to our hospital on Day 3 after her initial presentation to the outside hospital with continuing complaints of shortness of breath, though without chest pain or fever. Her past medical history was significant for multiple hospitalizations for pleuritis and pericaditis, most recently seven months prior for pericarditis. That episode had resolved after a three day course of pulse steroids. Vitals demonstrated a temperature of $98.4^{\circ} \mathrm{F}$, a pulse of $79 \mathrm{bpm}$, a respiratory rate of $20 / \mathrm{min}$, and a blood pressure of 118/70. Physical exam illustrated a rash, symmetrically distributed across the malar aspects of her face. Her exam including cardiac auscultation was reportedly benign. An echocardiogram showed the persistence of a small pericardial effusion and mild left atrial enlargement. A chest $\mathrm{x}$ ray revaled bilateral pulmonary edema with bibasilar atelectasis and cardiomegaly. EKG and laboratory tests were within normal limits except for an elevated ESR and CRP. The patient was promptly started on pulse steroids for three days.

Despite completing her course of steroids, repeat transthoracic echocardiography visualized persistence of a small pericardial effusion. A repeat chest CT to evaluate her pleural effusion imaged a $2.1 \mathrm{~cm}$ dense lesion in the right upper quadrant of her lung. PPD and an acid-fast bacilli (AFB) stain were negative. A bronchoalveolar lavage did not demonstrate any purulent secretions or notable AFB stains. Clinically, the patient had markedly improved, denying shortness of breath, chest pain, and cough. Nevertheless, empiric antibiotic therapy with vancomycin and piperacillin/tazobactam was initiated.

On the evening of Day 8, the patient began complaining of severe chest pain. On examination, she had visible jugular venous dilation to her mandible and pulsus paradoxus of approximately $10 \mathrm{~mm} \mathrm{Hg}$. She was tachycardic at 109 beats per minute, blood pressure was $92 / 60$, and her respiratory rate was $25 \mathrm{resps} / \mathrm{min}$. A stat echocardiogram revealed markedly increased pericardial fluid causing IVC collapse with mitral valve inflow respiratory variation. The right heart was not collapsed. EKG showed sinus tachycardia with T-wave flattening in V5, V6, and aVL. The patient was immediately taken to the cardiac catheterization lab for pericardiocentesis. A total of $680 \mathrm{cc}$ of yellow, purulent fluid was drained with staining revealing gram positive cocci in clusters which eventually grew methicillin-sensitive Staphylococcus Aureus (MSSA) on culture. Vancomycin was continued and piperacillin/tazobactam was discontinued. Two days later the patient received a pericardial window to prevent further episodes of effusion and tamponade. Vancomycin was initially narrowed to nafcillin but after developing a bout of acute renal failure this was changed to cefazolin. Despite a complicated hospital stay, she was discharged home in good health.

\section{Discussion}

Cardiac tamponade is a known complication of SLE, albeit a very rare manifestation. ${ }^{1}$ More typical cardiac manifestations of SLE include pericarditis and pericardial effusion, as seen in our patient, along with myocarditis, endocarditis, and conduction abnormalities. ${ }^{2}$ Causes of pericardial effusions include idiopathic, viral, bacterial, uremic, traumatic, or neoplastic pathologies. In connective tissue disorders, effusions are most typically caused by inflammatory cell infiltrate and immune complex deposition within cardiac tissue, causing a serous or serosanguanous exudate similar to synovial fluid to encompass the heart. ${ }^{3}$ This pericarditis and effusion can be significant, but rarely causes pericardial tamponade. ${ }^{3}$ When it does, it tends to occur in males later in life. ${ }^{4}$

While rare, it is not necessarily surprising that the patient presented here developed cardiac tamponade early in life. There are numerous published case reports which detail SLE patients developing tamponade, even as their presenting symptom. ${ }^{5,6}$ This case is noteworthy due to the bacterial etiology of her pericardial effusion \& tamponade. A similar case was presented by Knodell et al. in Chest 1974 which chronicled a female with known SLE who presented with fever, chills, and a significant pericardial effusion, but without positive blood cultures. ${ }^{7}$ It was only when thoracentesis revealed a Staphylococcal effusion that the source of her infection was found after steroid treatment on her $11^{\text {th }}$ day of hospitalization. The patient described in our case differs in that she remained afebrile throughout the course and did not show any tell-tale signs of infection until her chest CT revealed the cavitary lesion in her lung. It is unusual for such a significant infection to remain relatively asymptomatic throughout its course. The origins of our own patient's pericarditis remain 
elusive. Obviously, her lengthy course of steroids contributed to her infection, but no source could be identified apart from the loculated lesion in her lung.

The clinical course of this patient is unique as well. Pericarditis with effusion is typically treated very successfully with highdose steroids. This patient was on a full course of high-dose steroids, along with a three day course of pulse steroids. Though she initially improved clinically, she rapidly developed cardiac tamponade overnight. The dramatic exacerbation of her effusion could indicate the infection developed later in her course and caused the rapid progression of illness.

\section{References}

1. BERGEN SS Jr. Pericardial effusion, a manifestation of systemic lupus erythematosus. Circulation. 1960 Jul;22:144-50.

2. Hetjmancik, $\mathrm{M}$ et al. The cardiovascular manifestations of systemic lupus erythematosus. Am Heart J 1964;68:119-30.

3. Spondnick, D. "Pericardial Diseases." Braunwald: Heart Disease: A Textbook of

4. Cardiovascular Medicine, 6th ed, 2001: 1869-61.Baker, S et al. Late onset systemic lupus erythematosus. Am J Med 1979;66:727-32.

5. Castier, $\mathrm{M}$ et al. Cardiac tamponade in systemic lupus erythematosus: report of four cases. Arquivos Brasileros de Cardiologia 2000;75:446-8.

6. Pop, $\mathrm{C}$ et al. Pericardial tamponade unmasking systemic lupus erythematosus. Presse Medicale 2001;30:1257.

7. Knodell, R et al. Staphylococcal pericarditis in a patient with systemic lupus erythematosus.

8. Chest 1974;49:237-6. 